\title{
Evaluación Práctica de la Anatomía Basada en la Teoría de las Inteligencias Múltiples de Gardner, Carrera de Odontología de la Universidad de Antofagasta, Chile
}

\author{
Practical Evaluation of the Anatomy Based on the Theory of Multiple Intelligences \\ of Gardner, Odontology Career of the Antofagasta University, Chile
}

Fernando García-Hernández

GARCÍA-HERNÁNDEZ, F. Evaluación práctica de la Anatomía basada en la teoría de las inteligencias múltiples de Gardner, Carrera de Odontología de la Universidad de Antofagasta, Chile. Int. J. Morphol., 24(1):83-88, 2006.

RESUMEN: La evaluación constituye un proceso dinámico, continuo y de diagnóstico, inherente a la educación, que rebasa la simple calificación del rendimiento.

Para el proceso evaluativo deben usarse todos los métodos disponibles, no sólo como medio para determinar la aprobación de la asignatura sino que como recurso para medir el proceso de enseñanza-aprendizaje y, que a su vez, permita al docente comprobar, en forma objetiva, el cumplimiento de lo establecido en el programa, gracias al uso de los diversos medios pedagógicos de enseñanza.

Durante muchos años, la evaluación práctica anatómica consistió en la valoración de la disección de diversas regiones topográficas, interrogaciones orales sobre estructuras que se encontraban en dichas disecciones y en la realización de pruebas o tests en los cuales se hacían preguntas sobre aspectos teóricos vertidos en clases magistrales, demostraciones o mediante el uso de textos o atlas. En las últimas décadas muchos investigadores educacionales han propuesto diversas teorías del aprendizaje, con la finalidad de aprovechar al máximo el potencial intelectual de los estudiantes, siendo, en general, todas aplicables, válidas y muchas se potencian entre sí.

El presente trabajo, más que una investigación docente, pretende informar sobre la metodología evaluativa práctica basada en la teoría de las inteligencias múltiples de Howard Gardner, aplicada durante el aprendizaje práctico de la asignatura de Anatomía Humana para Odontología de la Universidad de Antofagasta, institución que ha implementado un modelo pedagógico centrado en el alumno y cuyos objetivos terminales se basan en el logro de competencias.

PALABRAS CLAVE: Educación; Anatomía; Inteligencias múltiples.

\section{INTRODUCCIÓN}

La evaluación debe ser un reflejo objetivo de los cambios que sufre el estudiante como de la enseñanza-aprendizaje en base a la conducta terminal que sea capaz de demostrar.

Es mediante la evaluación que se aprecia el logro de los objetivos programados en la asignatura. Por lo tanto, la evaluación debe estar en íntima relación con los objetivos propuestos (Comité de Expertos en Anatomía OPS/OMS, 1970; De Lara Galindo, 1971).

Para autores como De Lara Galindo (op. cit.) "la evaluación del aprendizaje debe ser constante; empezar antes de iniciarse el período lectivo, practicarse día a día, periódica, mensual, trimestralmente y al terminar el curso, recomendándose un examen totalizador". El mismo autor señala que "una de las razones por las cuales la evaluación ha de ser permanente, es porque el estudiante, sujeto de la educación, está sometido a un proceso incesante de modificaciones en virtud del aprendizaje".

Un efectivo método de evaluar la calidad de los estudiantes es mediante la medición de sus habilidades por examinaciones periódicas tanto teóricas como prácticas (DiDio, 1971).

Unidad de Anatomía, Departamento Biomédico, Facultad de Ciencias de la Salud, Universidad de Antofagasta, Chile.

Patrocionio Dirección de Docencia, Vicerrectoría Académica, Universidad de Antofagasta, Proyecto de Docencia Nº 1604. 
Para el proceso evaluativo deben usarse todos los métodos disponibles, no sólo como medio para determinar la aprobación de la asignatura, sino que como recurso para medir el proceso de enseñanza-aprendizaje y que a su vez le permita al docente comprobar, en forma objetiva, el cumplimiento de lo establecido en el programa gracias al uso de los diversos medios pedagógicos de enseñanza (García, 1970).

Se recomienda que la evaluación práctica reúna las siguientes características: ser objetiva, ser completa, ser breve y ser práctica (Rodríguez \& Ceppi, 1983).

Guiraldes (1983), nos indica que todo objetivo debe ser evaluado y el resultado de esta evaluación conocido por el estudiante en la forma más rápida posible. La comprobación rápida de resultados positivos estimula el interés por aprender y el conocimiento tiende a ser fijado. Por otra parte, un resultado negativo conocido a tiempo otorga la posibilidad de recapitular.

Rodríguez \& Fernández (1985) señalan que la evaluación del aprendizaje centra su preocupación en los resultados que el docente desea que se logren: objetivos y a la vez conductas.

Se ha comprobado que los estudiantes prefieren realizar evaluaciones intermedias o clase a clase, que la realización de una sola evaluación final al terminar un capítulo o unidad temática (Matamala et. al., 1985).

Para las evaluaciones en Anatomía deben preferirse los controles escritos, incluyéndose preguntas de respuestas abierta y fija con sus respectivas variantes, destacando las evaluaciones objetivas y disminuyendo el azar al mínimo. Esta evaluación deberá ser creíble, comparable, válida y discriminativa (DiDio, 1973).

El uso de imágenes en softwares docentes o mediante otro tipo de sistemas, sirven como sistema de repaso o de preparación de la actividad práctica, pero no reemplazan, según los propios estudiantes al paso práctico, la clase teórica y las lecturas programadas de textos (Insunza \& Bravo, 1999). Al realizarse comparaciones evaluativas se comprobó un mejor rendimiento en los alumnos que utilizaron software unido al paso práctico, respecto de otras combinaciones metodológicas (clase más software). La actividad práctica es ineludible e irremplazable para la docencia de la Anatomía (Inzunza \& Bravo).

Se entiende que la evaluación es parte de un proceso integral, donde mediante diversos medios se pretende averiguar en forma global el nivel de los aprendizajes sig- nificativos por los alumnos y determinar cuali y cuantitativamente los conocimientos, teóricos y prácticos adquiridos por los alumnos (Barrovecchio et al., 2001).

Es casi imposible concebir un proceso de aprendizaje sin evaluación y menos aún no evaluar las acciones de enseñanza y educativas (Barrovecchio et al.).

La evaluación forma parte integral del proceso educativo en todas sus etapas y en todos sus aspectos (Tartarini, 1967). La evaluación constituye un proceso dinámico, continuo y de diagnóstico, inherente a la educación, que rebasa la simple calificación del rendimiento (Tartarini).

La evaluación es una necesidad en educación. La evaluación es tan vieja como el proceso educativo. La evaluación retroalimenta y orienta la tarea docente. La evaluación es parte del proceso educativo, debe ser continua y estar de acuerdo a los objetivos de enseñanza programados (De Zavaleta, 1971). La evaluación no debe limitarse sólo a constatar la memorización de los contenidos del programa, sino que debe medir el logro completo de todos los objetivos establecidos en el programa (De Zavaleta).

Howard Gardner (1994), autor de la teoría de las inteligencias múltiples, señala que la inteligencia es un conjuntos de capacidades que permiten al sujeto solucionar problemas y a la vez, generar productos considerados importantes en un determinado contexto cultural. También señala que las mal llamadas pruebas objetivas son un freno para el desarrollo del entedimiento, debiendo realizarse "evaluaciones auténticas" que corresponden a aquellas basadas en los desempeños. Así, un estudiante de Odontología, futuro Cirujano Dentista, debe tener desarrolladas, en mayor o menor grado, diversas inteligencias: lingüística, lógica-matemática, espacial, cinestésico-corporal, naturalista, interpersonal e intrapersonal. La evaluación debe estar en coherencia con el trabajo que el docente realiza en el aula o en cualquier otro espacio educativo. Debe abarcar todos los aspectos de la cognición y dar la posibilidad a cada alumno de mostrar y demostrar sus capacidades en estas distintas áreas y no sólo privilegiar los aspectos lingüisticos o lógico-matemáticos. Hay que atender y respetar las diferencias individuales de los estudiantes: educación centrada en el individuo que lo habilite para su permanente autoaprendizaje y autonomía (Gardner).

Daniel Goleman (1997), al formular la teoría de la inteligencia emocional, señaló que las emociones forman parte importante de la vida psicológica del estudiante y que tienen una alta influencia en la motivación académica y en las estrategias cognitivas (adquisición, almacenamiento, recuperación de la información, etc.) y, por ende, en el 
aprendizaje y en el rendimiento escolar (Pekrum, 1992). El impacto de las emociones sobre el aprendizaje y el rendimiento es muy importante; existen emociones positivas de la tarea que motivan y conducen a un incremento del rendimiento (disfrutar de la tarea) y emociones negativas de la tarea, que tienen efectos globales, pudiendo ser positivas o negativas, tales como aburrimiento, ansiedad (que puede provocar alta motivación) o insatisfacción.

Por medio de la evaluación determinamos el valor o el grado de eficiencia de algún proceso de enseñanza o de alguna experiencia de aprendizaje. Evaluación es el proceso de valorar cambios de conducta al término de los objetivos que se persiguen (Ahumada, 1972). La evaluación debe reunir, organizar, analizar y comunicar la información que es necesaria para la toma de decisiones. El proceso de evaluación será efectivo en la medida que facilita una información adecuada (validez), confiable (confiabilidad) y en el momento que es requerida (temporalidad).

Para los estudios anatómicos en Odontología deben desarrollarse una Anatomía Descriptiva tanto de la boca y del sistema estomatognático como del organismo en general, fundamentales para la comprensión científica del cuerpo humano, por y para el profesional Odontólogo que forma parte del equipo de salud multidisciplinario; y una Anatomía Aplicada enfatizando aquellos conceptos anatómicos fundamentales para la práctica de la profesión odontológica. Las actividades prácticas deben ser todas evaluadas y considerar especial atención en la disección de regiones bucodentarias, extrapolar a técnicas propias de la morfología odontológica (dibujo, modelado y tallado), imagenería diagnóstica, seminarios teórico-prácticos trabajos monográficos, nuevas metodologías didácticas: multimedia, autoaprendizaje, autoevaluación, realidad virtual, etc. (Sociedad Anatómica Española, 1996).

Durante muchos años, la evaluación práctica anatómica consistió en la valoración de la disección de diversas regiones topográficas, interrogaciones orales sobre estructuras que se encontraban en dichas disecciones y en la realización de pruebas o tests en los cuales se hacían preguntas sobre aspectos teóricos vertidos en clases magistrales, demostraciones, o mediante el uso de textos o atlas.

En las últimas décadas muchos investigadores educacionales (Piaget, Novak, Ausubel, Hanesian, Gowin, Vygotsky, Waldsworth, Gardner, Goleman entre otros) han propuesto diversas teorías del aprendizaje, con la finalidad de aprovechar al máximo el potencial intelectual de los estudiantes, siendo, en general, todas aplicables, válidas y muchas se potencian entre sí.
El presente trabajo, más que una investigación docente, pretende informar sobre la metodología evaluativa práctica utilizada en la asignatura de Anatomía Humana, para Odontología de la Universidad de Antofagasta, con la finalidad de aplicar, aunque en forma primaria, la teoría de las inteligencias múltiples de Howard Gardner al aprendizaje práctico de la disciplina.

\section{METODOLOGÍA EVALUATIVA UTILIZADA}

Las actividades de laboratorio de la asignatura son evaluadas utilizando algunos de los siguientes aspectos metodológicos:

1) Evaluación de inicio de laboratorio, que permite determinar los conocimientos teóricos que posee cada alumno respecto del o los temas a tratar en el práctico. Se publica la pauta de respuestas correctas y una vez corregida y calificada se entrega a cada alumno para la identificación de éxitos y errores para permitir la retroalimentación. Mide las inteligencias lingüística y lógico-matemática.

2) Evaluación de término de laboratorio, a través de la cual se obtiene el grado de aprendizaje, mediante la identificación de estructuras gracias a la utilización de preparados anatómicos en formol o momificados, o, en su defecto, láminas o dibujos. Para evaluar el aprendizaje durante el laboratorio, se usan los mismos preparados anatómicos, láminas y/o dibujos del laminario anatómico, dibujos y fotografías de atlas, programas computacionales, etc. También se publica la pauta de respuestas correctas, entregándose corregida y calificada a cada alumno para la identificación de éxitos y errores, con el objeto de permitir la retroalimentación. Mide inteligencias lingüística y lógicomatemática, pero, primordialmente, las inteligencias naturalista, cinestésico-corporal y espacial.

3) Evaluación teórico-práctica de temas específicos, la cual se efectúa transcurrido un breve tiempo desde la realización del o de los laboratorios que se evalúan, favoreciendo el reciclaje y repaso de conocimientos y conceptos. Pruebas de este tipo son aplicadas cuando no están programadas en la asignatura evaluaciones de inicio y/o de término. Mide las inteligencias lingüística y lógicomatemática.

4) Control práctico final de Unidad, mediante la identificación de estructuras anatómicas en preparados, modelos, radiografías y/o láminas (García-Hernández, 2003). Mide fundamentalmente las inteligencias espacial, naturalista y cinestésico-corporal. 
5) Tallado de macro modelo anatómico topográfico Mide las inteligencias espacial, naturalista y cinestésico-corporal.

6) Tallado de macro modelo de anatomía dentaria Mide las inteligencias espacial, naturalista y cinestésico-corporal.

7) Seminario de investigación grupal, revisión bibliográfica y de aprendizaje basado en la resolución de problemas (ABRP). Mide en forma prioritaria las inteligencias lingüística, lógico-matemática, naturalista e interpersonal.

8) Defensa grupal de investigación, revisión bibliográfica y de aprendizaje basado en la resolución de problemas (ABRP), Mide las inteligencias lingüística, lógico-matemática, naturalista, interpersonal e intrapersonal (para quienes deben exponer antes sus pares).

9) Poster o panel de exposición pública de temas específicos de trabajo individual, donde se debe describir, en forma breve, alguna característica anatómica normal o patológica de estructuras anatómicas, descripción apoyada por dibujos realizados por el alumno. Mide las inteligencias lingüística, lógico-matemática, espacial, naturalista e intrapersonal (ya que representa una interpretación propia, individual de una situación general).

10) Trabajo personal de programas computacionales en Laboratorio de Multimedios. Mide primordialmente las inteligencias lógico-matemática, espacial, naturalista e intrapersonal.

11) Laminario anatómico. Mide fundamentalmente las inteligencias lingüística, lógico-matemática, espacial, naturalista e intrapersonal.

Por lo tanto, en la evaluación práctica de la asignatura de Anatomía se están aplicando 7 de las 8 inteligencias propuestas por Gardner. Sólo se está excluyendo la inteligencia musical y, de acuerdo a las últimas propuestas del autor, la inteligencia existencial, aunque el mismo Gardner duda de la evidencia cerebral de una inteligencia existencial.

\section{DISCUSIÓN}

A partir del año 2004, la Universidad de Antofagasta está aplicando un modelo pedagógico centrado en el estudiante que enfatiza en el logro de competencias finales.

Se puede definir competencia como aquellas habilidades que tiene que dominar todo profesional y que emanan del ejercicio de su profesión, debiendo incluir aspectos cognitivos, técnico-profesionales y actitudinales.
La definición de Kane (1992) en Brailovsky (2002) parece representar una de las definiciones más dinámicas e interesantes. Este autor define competencia profesional como: "... el grado de utilización de los conocimientos, las habilidades y el buen juicio asociados a la profesión, en todas las situaciones que se pueden confrontar en el ejercicio de la práctica profesional".

Las competencias y habilidades que debe poseer un Cirujano Dentista General dependerán de las exigencias que le exija la comunidad para su desempeño profesional en los ámbitos público y privado.

Cada institución de Educación Superior debe definir el perfil profesional que desea que sus egresados posean al salir al medio laboral, y que, de alguna manera, representa la impronta formativa lograda al finalizar sus estudios de pregrado.

La Universidad de Antofagasta ha establecido las competencias generales que deben lograr sus estudiantes y, a su vez, cada Carrera ha determinado las competencias profesionales específicas, según el perfil profesional de egreso y donde cada asignatura aporta las habilidades y conocimientos necesarios para el logro de dichas competencias.

De las competencias generales propuestas por la Universidad, la asignatura de Anatomía aplica fundamentalmente comunicación, pensamiento crítico y autoaprendizaje

En tanto, del análisis del perfil de la Carrera, la asignatura de Anatomía pudo extrapolar las competencias anatómicas que debe poseer un estudiante de pregrado de Odontología al finalizar la asignatura de Anatomía Humana, las cuales son:

1) Manejo de la Nomenclatura anatómica actual para:

a) Interrelación y entendimiento con sus pares.

b) Uso adecuado de términos y conceptos aplicables para la elaboración de una historia clínica.

c) Interpretación del lenguaje técnico aportado por la literatura científica básica y especializada.

2) Manejo de conceptos primordiales de anatomía sistémica y neuroanatomía para la comprensión de las otras disciplinas básicas (histología, fisiología, patología, etc.).

3) Acabado conocimiento descriptivo de los órganos que estructuran al sistema estomatognático, sus relaciones entre sí y su aplicación en relación a las asignaturas clínicas, en lo que compete a:

a) Técnicas anestésicas.

b) Procedimientos clínicos.

c) Imagenología. 
4) Colaborar en la capacitación del estudiante para la educación continuada, mediante el aprendizaje autodirigido, la autoevaluación y la elaboración de miniproyectos de revisión bibliográfica y de trabajo personal.

La utilización de diversos métodos de enseñanzaaprendizaje durante el aprendizaje práctico de la Anatomía, basados en las diferentes inteligencias que plantea Gardner en su teoría, nos ha demostrado que incrementa los parámetros válidos para educar y para evaluar el logro de las competencias anatómicas necesarias para el desarrollo posterior de las competencias profesionales.

\section{CONCLUSIONES}

1. El aprendizaje práctico en el Laboratorio de Anatomía debe ser multifactorial, utilizándose la mayor cantidad de variables que permitan aprovechar al máximo las diversas in- teligencias que posee cada estudiante.

2. Las nuevas tecnologías de la información y comunicación (TICs) deben ser aplicadas constantemente, permitiendo el trabajo directo durante el Laboratorio Práctico y muy en especial como actividad ex-aula.

3. Sería recomendable aplicar un test de inteligencias múltiples al iniciar la actividades lectivas de la asignatura, como una forma de identificar las diversas inteligencias que poseen los estudiantes al empezar su Carrera.

4. Siendo la Anatomía una disciplina cuya enseñanzaaprendizaje durante la Educación Media es mínima y de poca profundidad, situación que dificulta su inmediato aprendizaje en la Educación Superior, creemos necesario que al inicio de la asignatura se desarrolle un proceso remedial de preparación especial que facilite el posterior aprendizaje de la disciplina.

GARCÍA-HERNÁNDEZ, F. Practical evaluation of the Anatomy based on the theory of multiple intelligences of Gardner, Odontology Career of the Antofagasta University, Chile. Int. J. Morphol., 24(1):83-88, 2006.

SUMMARY:The evaluation constitutes a dynamic, continuous process of diagnosis, inherent to the education, that exceeds the simple qualification of the yield.

For the evaluation process all the methods available must be used, non only as a mean to determining the approval of the subject but as a resource to measure the education-learning process. Thus, the teacher may verify, in objective form, the fulfillment of established in the program thanks to the use of diverse pedagogical means of education. For many years, the anatomical practical evaluation consisted of the valuation of the dissection of diverse topographic regions, oral interrogations on structures that were in these dissections, and the accomplishment of tests in which questions became on spilled theoretical aspects in theoretical classes, demonstrations or by means of the use of texts or atlas.

In the last decades, many educational investigators have proposed diverse learning theories, with the purpose of taking advantage to the maximum the intellectual potential of the students. In general, all of them have been applicable, valid and many are harnessed to each other.

The present work, more than an educational investigation, tries to inform on the practical evaluation method based upon the Howard Gardner's multiple intelligences theory, applied during the practical learning of the subject of Human Anatomy for Dentistry of the University of Antofagasta, institution that has implemented a centred pedagogical model in the student and whose terminal objectives are based on the profit of competences.

KEY WORDS: Education; Anatomy; Multiple intelligences.

\section{REFERENCIAS BIBLIOGRÁFICAS}

Ahumada, P. Apuntes Docentes Curso Problemática en Evaluación Universitaria. Consejo Coordinador Universitario de Valparaíso, Universidades de Chile, Técnica Federico Santa María y Católica de Valparaíso, 1972.

Barrovecchio, J. C.; Pérez, B.; Bella de Paz, L.; Busmail, L. \& Ruggero, E. Evaluación de los exámenes de Anatomía Normal en la Universidad Nacional de Rosario. Rev. Chil. Anat. 19(2):139-44, 2001.
Brailovsky, C. A. Educación Médica, evaluación de las competencias, 2000. http://www.fmv.uba.org.ar/proaps/9pdf

Comité de Expertos de la OMS/OPS en la Enseñanza de la Morfología. Enseñanza de la Morfología en las Escuelas de Medicina de la América Latina. Primer Informe. Serie Desarrollo de Recursos Humanos A. Educación Médica No 9, OPS, Oficina Sanitaria Panamericana, Oficina Regional de la OMS, Washington D. C., 1970. 
De Lara Galindo, S. Evaluación del aprendizaje. Arch. Mex. Anat., 35(11):31-45, 1971.

De Zavaleta, E.T. Evaluación de materiales audiovisuales para la enseñanza. EUDEBA, Buenos Aires, 1971.

DiDio, L. J. A. Systems of teaching anatomy and evaluation of students. Arch. Mex. Anat., 36(11):23-43, 1971.

DiDio, L. J. A. Tendencias modernas en la educación médica y su repercusión en la enseñanza de la Anatomía. Educación médica y salud, 2:184-97, 1973.

García R. M. Relato del III Seminario y III Congreso Centroamericano de Anatomía en la ciudad de Guatemala y del I Venezolano y II Panamericano en Venezuela. Arch. Mex. Anat., 34(10):7-10, 1970.

Gardner, H. Estructuras de la mente. La teoría de las inteligencias múltiples. $2^{\mathrm{a}}$ ed. Fondo de la Cultura Económica, Ciudad de México, 1994.

García-Hernández, F. Evaluación del aprendizaje práctico de la Anatomía Humana para Odontología en la Universidad de Antofagasta, Chile. Int. J. Morphol., 21(1):4347, 2003.

Goleman, D. La inteligencia emocional. Javier Vergara Editor, S. A., Buenos Aires, 1997.

Guiraldes, H. Módulos de Aprendizaje en Anatomía Clínica. An. Anat. Nor., 1:140-2, 1983.

Insunza, O. \& Bravo, H. Impacto de dos programas computacionales de Anatomía Humana en el rendimiento del conocimiento práctico de los alumnos. Rev. Chil. Anat. 17(2):205-9, 1999.

Matamala, F.; Cabalin, D. \& Fuentes, J. Evaluación de un curso de Anatomía nivel A por los alumnos. An. Anat. Nor., 3:64-7, 1985.

Pekrum, R. The impact of emotions on learning an achievement, in Applied Psichology. An international review 41(4):359-76, 1992.

Rodríguez,A \& Ceppi, H. Metódica de Control de Trabajos Prácticos en Anatomía Normal para alumnos del Primer Año de Medicina. An. Anat. Nor. 1:135-6, 1983.

Rodríguez, A \& Fernández, S. Prospección en la dispersión de las preguntas de acuerdo al uso de un banco de preguntas calificadas. An. Anat. Nor., 3:87-9, 1985.
Sociedad Anatómica Española. Bases para los programas en los planes de estudio de las diferentes Licenciaturas y Diplomaturas. Patrocinado por Sociedad Anatómica Española. II Jornadas sobre Enseñanza de la Anatomía. Universidad Complutense, Facultad de Medicina, Madrid 10 de Mayo de 1996.

Tartarini, E. Evaluación escolar y elementos de estadística aplicada. $2^{\text {a }}$ Reimpresión. Editorial Universitaria, Santiago, 1967.

Dirección para correspondencia: Prof. Dr. Fernando Garcia-Hernández

Unidad de Anatomía

Departamento Biomédico

Facultad de Ciencias de la Salud

Universidad de Antofagasta

CHILE

Recibido : 28-12-2005

Aceptado: 25-01-2006

Email:fgarcia@uantof.cl 